\title{
Insight into the Relationship between Viscosity and \\ Hydrogen Bond of a series of Imidazolium Ionic Liquids: a Molecular Dynamics and Density Functional Theory Study
}

\author{
Kun Jiang a,b, Lei Liu ${ }^{a}$, Xiaomin Liu ${ }^{c}$, Xiaochun Zhang a, Suojiang Zhang ${ }^{a^{*}}$
}

${ }^{a}$ CAS, Beijing Key Laboratory of Ionic Liquids Clean Process, Key Laboratory of Green Process and Engineering, State Key Laboratory of Multiphase Complex System, Institute of Process Engineering, Chinese Academy of Sciences, Beijing, 100190, China

${ }^{b}$ College of Chemical and Engineering, University of Chinese Academy of Sciences, Beijing, 100049, China

c School of Chemistry and Chemical Engineering, Qingdao University, Qingdao 266071, Shandong, China

* Corresponding author. Tel./fax: +86-010-82544875.

E-mail addresses: sjzhang@ipe.ac.cn (S. J. Zhang); 
Table S1. Calculated and experimental ${ }^{1,2}$ value of densities and viscosities of three ILs

\begin{tabular}{|c|c|c|c|c|c|c|}
\hline $\begin{array}{c}T \\
(\mathrm{~K})\end{array}$ & $\begin{array}{c}\rho_{\text {sim }} \\
\left(\mathrm{g} / \mathrm{cm}^{3}\right)\end{array}$ & $\begin{array}{c}\rho_{\exp } \\
\left(\mathrm{g} / \mathrm{cm}^{3}\right)\end{array}$ & Error & $\begin{array}{c}\eta_{\text {sim }} \\
(\mathrm{mPa} \cdot \mathrm{s})\end{array}$ & $\begin{array}{c}\eta_{\exp } \\
(\mathrm{mPa} \cdot \mathrm{s})\end{array}$ & Error \\
\hline \multicolumn{7}{|c|}{$[\mathrm{Bim}]\left[\mathrm{NTf}_{2}\right]$} \\
\hline 293 & $1.5126 \pm 0.0031$ & 1.4799 & $2.20 \%$ & $136.36 \pm 10.28$ & 129.14 & $5.59 \%$ \\
\hline 303 & $1.5015 \pm 0.0031$ & 1.4701 & $2.14 \%$ & $79.797 \pm 6.826$ & 71.370 & $11.81 \%$ \\
\hline 313 & $1.4913 \pm 0.0033$ & 1.4603 & $2.12 \%$ & $51.832 \pm 2.264$ & 47.138 & $9.96 \%$ \\
\hline 323 & $1.4815 \pm 0.0034$ & 1.4505 & $2.13 \%$ & $33.273 \pm 1.124$ & 33.301 & $-0.08 \%$ \\
\hline 333 & $1.4719 \pm 0.0035$ & 1.4409 & $2.16 \%$ & $24.113 \pm 0.218$ & 24.075 & $0.16 \%$ \\
\hline 343 & $1.4624 \pm 0.0036$ & 1.4313 & $2.17 \%$ & $17.404 \pm 0.25$ & 18.186 & $-4.30 \%$ \\
\hline \multicolumn{7}{|c|}{$[\mathrm{Bmim}]\left[\mathrm{NTf}_{2}\right]$} \\
\hline 293 & $1.4643 \pm 0.0030$ & 1.4410 & $1.62 \%$ & $67.394 \pm 7.765$ & 60.659 & $11.10 \%$ \\
\hline 303 & $1.4547 \pm 0.0030$ & 1.4314 & $1.63 \%$ & $42.964 \pm 4.652$ & 39.543 & $8.65 \%$ \\
\hline 313 & $1.4454 \pm 0.0031$ & 1.4219 & $1.65 \%$ & $28.414 \pm 1.830$ & 27.252 & $4.26 \%$ \\
\hline 323 & $1.4352 \pm 0.0032$ & 1.4124 & $1.62 \%$ & $20.076 \pm 1.400$ & 19.729 & $1.76 \%$ \\
\hline 333 & $1.4252 \pm 0.0032$ & 1.4030 & $1.58 \%$ & $15.381 \pm 0.966$ & 15.198 & $1.20 \%$ \\
\hline 343 & $1.4159 \pm 0.0033$ & 1.3937 & $1.59 \%$ & $11.499 \pm 0.501$ & 11.724 & $-1.92 \%$ \\
\hline \multicolumn{7}{|c|}{$[\mathrm{Bmmim}]\left[\mathrm{NTf}_{2}\right]$} \\
\hline 293 & $1.4294 \pm 0.0029$ & 1.4235 & $0.41 \%$ & $129.78 \pm 10.10$ & 131.90 & $-1.61 \%$ \\
\hline 303 & $1.4211 \pm 0.0029$ & 1.4142 & $0.49 \%$ & $77.183 \pm 6.288$ & 77.731 & $-0.71 \%$ \\
\hline 313 & $1.4113 \pm 0.0029$ & 1.4049 & $0.45 \%$ & $44.184 \pm 1.320$ & 49.703 & $-11.10 \%$ \\
\hline 323 & $1.4014 \pm 0.0032$ & 1.3957 & $0.41 \%$ & $29.878 \pm 0.657$ & 33.720 & $-11.39 \%$ \\
\hline 333 & $1.3915 \pm 0.0032$ & 1.3866 & $0.35 \%$ & $21.303 \pm 0.581$ & 23.984 & $-11.18 \%$ \\
\hline 343 & $1.3824 \pm 0.0033$ & 1.3775 & $0.35 \%$ & $15.660 \pm 0.147$ & 17.993 & $-12.97 \%$ \\
\hline
\end{tabular}

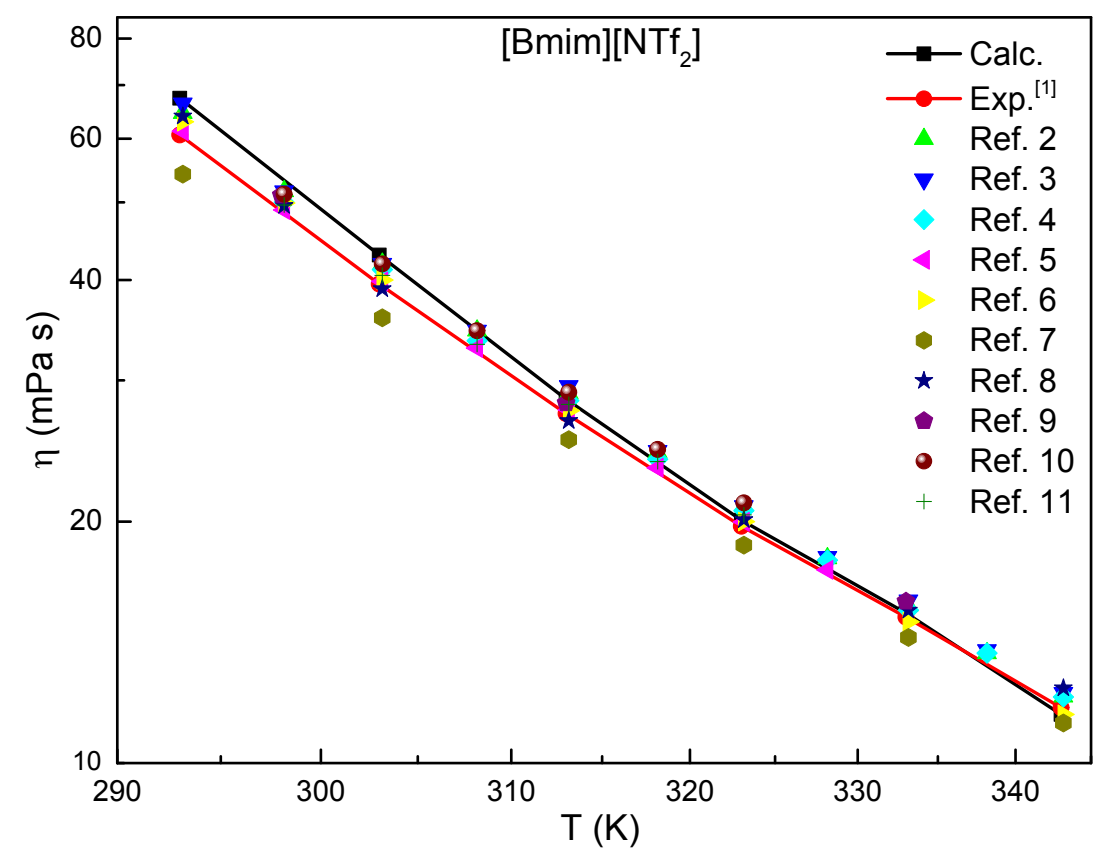

Figure S1. Calculated viscosities of ionic liquids $[\mathrm{Bmim}]\left[\mathrm{NTf}_{2}\right]$ as a function of temperature. Available experimental results ${ }^{1,3-12}$ are included for comparison. 


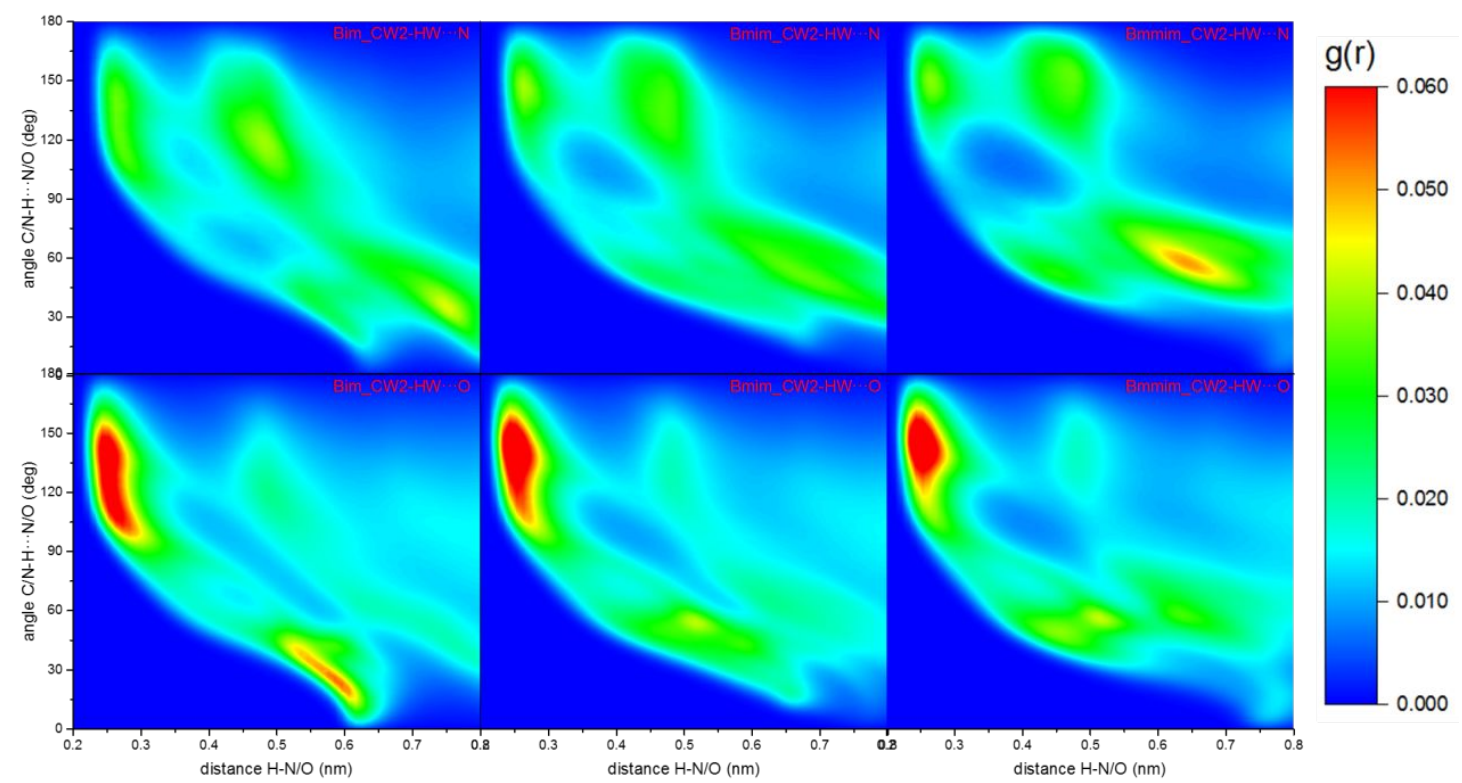

Figure S2. Combined distribution functions of hydrogen bond between the $\mathrm{C} 5-\mathrm{H}$ site of imidazolium ring of cations and $\mathrm{N} / \mathrm{O}$ atoms of anions in the three ionic liquids.

Table S2. Number of hydrogen bond of ILs at different temperatures.

\begin{tabular}{cccccccc}
\hline ILs & Donor & $\mathbf{2 9 3} \mathbf{~ K}$ & $\mathbf{3 0 3} \mathbf{~ K}$ & $\mathbf{3 1 3} \mathbf{~ K}$ & $\mathbf{3 2 3} \mathbf{~ K}$ & $\mathbf{3 3 3} \mathbf{K}$ & $\mathbf{3 4 3} \mathbf{~ K}$ \\
\hline BimNTf & $\mathrm{N}_{1}-\mathrm{HN}$ & $2.50 \pm 0.032$ & $2.46 \pm 0.032$ & $2.45 \pm 0.033$ & $2.43 \pm 0.033$ & $2.41 \pm 0.033$ & $2.39 \pm 0.034$ \\
& $\mathrm{CR}-\mathrm{HR}$ & $1.96 \pm 0.034$ & $1.93 \pm 0.033$ & $1.90 \pm 0.035$ & $1.88 \pm 0.033$ & $1.85 \pm 0.034$ & $1.83 \pm 0.034$ \\
& $\mathrm{CW}_{1}-\mathrm{HW}$ & $1.78 \pm 0.034$ & $1.74 \pm 0.035$ & $1.72 \pm 0.036$ & $1.70 \pm 0.035$ & $1.67 \pm 0.036$ & $1.66 \pm 0.035$ \\
& $\mathrm{CW}_{2}-\mathrm{HW}$ & $1.61 \pm 0.030$ & $1.57 \pm 0.032$ & $1.55 \pm 0.032$ & $1.53 \pm 0.033$ & $1.51 \pm 0.032$ & $1.48 \pm 0.032$ \\
BmimNTf $_{2}$ & $\mathrm{CR}-\mathrm{HR}$ & $1.91 \pm 0.032$ & $1.88 \pm 0.034$ & $1.85 \pm 0.032$ & $1.83 \pm 0.033$ & $1.80 \pm 0.032$ & $1.78 \pm 0.032$ \\
& $\mathrm{CW}_{1}-\mathrm{HW}$ & $1.54 \pm 0.032$ & $1.51 \pm 0.032$ & $1.49 \pm 0.031$ & $1.47 \pm 0.032$ & $1.45 \pm 0.031$ & $1.43 \pm 0.032$ \\
& $\mathrm{CW}_{2}-\mathrm{HW}$ & $1.50 \pm 0.031$ & $1.48 \pm 0.032$ & $1.45 \pm 0.031$ & $1.43 \pm 0.032$ & $1.41 \pm 0.031$ & $1.38 \pm 0.031$ \\
BmmimNTf $_{2}$ & $\mathrm{CW}_{1}-\mathrm{HW}$ & $1.40 \pm 0.031$ & $1.38 \pm 0.030$ & $1.37 \pm 0.031$ & $1.35 \pm 0.032$ & $1.33 \pm 0.031$ & $1.31 \pm 0.031$ \\
& $\mathrm{CW}_{2}-\mathrm{HW}$ & $1.36 \pm 0.029$ & $1.34 \pm 0.029$ & $1.32 \pm 0.030$ & $1.30 \pm 0.031$ & $1.28 \pm 0.030$ & $1.25 \pm 0.031$ \\
\hline
\end{tabular}

Table S3. Interaction energy of hydrogen bond of ILs at different temperatures $\left(\mathrm{kJ} \cdot \mathrm{mol}^{-1}\right)$

\begin{tabular}{cccccccc}
\hline ILs & Donor & $\mathbf{2 9 3} \mathbf{K}$ & $\mathbf{3 0 3} \mathbf{K}$ & $\mathbf{3 1 3} \mathbf{K}$ & $\mathbf{3 2 3} \mathbf{K}$ & $\mathbf{3 3 3} \mathbf{K}$ & $\mathbf{3 4 3} \mathbf{K}$ \\
\hline \multirow{2}{*}{ BimNTf $_{2}$} & $\mathrm{~N}_{1}$-HN & $-121.07 \pm 1.38$ & $-119.31 \pm 1.39$ & $-118.4 \pm 1.43$ & $-117.16 \pm 1.44$ & $-116.10 \pm 1.45$ & $-115.12 \pm 1.5$ \\
& $\mathrm{CR}-\mathrm{HR}$ & $-62.95 \pm 1.07$ & $-61.78 \pm 1.06$ & $-60.93 \pm 1.12$ & $-60.10 \pm 1.06$ & $-59.21 \pm 1.10$ & $-58.39 \pm 1.10$ \\
& $\mathrm{CW}_{1}$-HW & $-54.86 \pm 1.06$ & $-53.55 \pm 1.07$ & $-53.00 \pm 1.13$ & $-52.31 \pm 1.08$ & $-51.34 \pm 1.11$ & $-50.88 \pm 1.10$ \\
& $\mathrm{CW}_{2}$-HW & $-14.74 \pm 0.32$ & $-14.42 \pm 0.31$ & $-14.19 \pm 0.32$ & $-13.98 \pm 0.33$ & $-13.71 \pm 0.32$ & $-13.49 \pm 0.32$ \\
& $\mathrm{CR}-\mathrm{HR}$ & $-39.35 \pm 0.65$ & $-38.78 \pm 0.70$ & $-38.16 \pm 0.65$ & $-37.69 \pm 0.68$ & $-37.06 \pm 0.66$ & $-36.53 \pm 0.66$ \\
BmimNTf $_{2}$ & $\mathrm{CW}$-HW & $-29.77 \pm 0.62$ & $-29.14 \pm 0.62$ & $-28.65 \pm 0.61$ & $-28.28 \pm 0.63$ & $-27.86 \pm 0.61$ & $-27.34 \pm 0.62$ \\
& $\mathrm{CW}_{2}$-HW & $-15.61 \pm 0.34$ & $-15.37 \pm 0.36$ & $-15.03 \pm 0.34$ & $-14.77 \pm 0.35$ & $-14.47 \pm 0.34$ & $-14.18 \pm 0.34$ \\
& $\mathrm{CW}_{1}$-HW & $-16.47 \pm 0.38$ & $-22.76 \pm 0.50$ & $-39.22 \pm 0.63$ & $-16.07 \pm 0.37$ & $-22.37 \pm 0.50$ & $-38.44 \pm 0.59$ \\
& $\mathrm{CW}_{2}$-HW & $-15.95 \pm 0.37$ & $-21.95 \pm 0.51$ & $-37.90 \pm 0.62$ & $-15.63 \pm 0.39$ & $-21.46 \pm 0.52$ & $-37.09 \pm 0.63$ \\
\hline
\end{tabular}


Table S4. Lifetime of hydrogen bond of ILs at different temperatures (ns)

\begin{tabular}{cccccccc}
\hline ILs & Donor & $\mathbf{2 9 3} \mathbf{~ K}$ & $\mathbf{3 0 3} \mathbf{~ K}$ & $\mathbf{3 1 3} \mathbf{~}$ & $\mathbf{3 2 3} \mathbf{~}$ & $\mathbf{3 3 3} \mathbf{~}$ & $\mathbf{3 4 3} \mathbf{~}$ \\
\hline \multirow{2}{*}{ BimNTf $_{2}$} & $\mathrm{~N}_{1}-\mathrm{HN}$ & 3.00 & 1.89 & 1.31 & 0.94 & 0.69 & 0.53 \\
& $\mathrm{CR}-\mathrm{HR}$ & 1.82 & 1.20 & 0.88 & 0.67 & 0.51 & 0.39 \\
& $\mathrm{CW}_{1}-\mathrm{HW}$ & 1.88 & 1.19 & 0.81 & 0.59 & 0.44 & 0.33 \\
& $\mathrm{CW}_{2}-\mathrm{HW}$ & 1.65 & 1.05 & 0.74 & 0.56 & 0.42 & 0.32 \\
BmimNTf & $\mathrm{CR}-\mathrm{HR}$ & 1.51 & 1.08 & 0.77 & 0.60 & 0.43 & 0.33 \\
& $\mathrm{CW}_{1}-\mathrm{HW}$ & 1.14 & 0.78 & 0.53 & 0.40 & 0.29 & 0.22 \\
& $\mathrm{CW}_{2}-\mathrm{HW}$ & 1.11 & 0.77 & 0.55 & 0.42 & 0.30 & 0.23 \\
BmmimNTf $_{2}$ & $\mathrm{CW}_{1}-\mathrm{HW}$ & 1.55 & 0.94 & 0.68 & 0.46 & 0.34 & 0.25 \\
& $\mathrm{CW}_{2}-\mathrm{HW}$ & 1.80 & 1.08 & 0.77 & 0.52 & 0.39 & 0.28 \\
\hline
\end{tabular}

The approximation of the time integral of correlation function we used refers to the previous study ${ }^{13}$. Assuming that the $\mathrm{C}(\mathrm{t})$ function decays as a pure exponential after $\mathrm{t}_{0}$. The schematic and derivation process are as following.

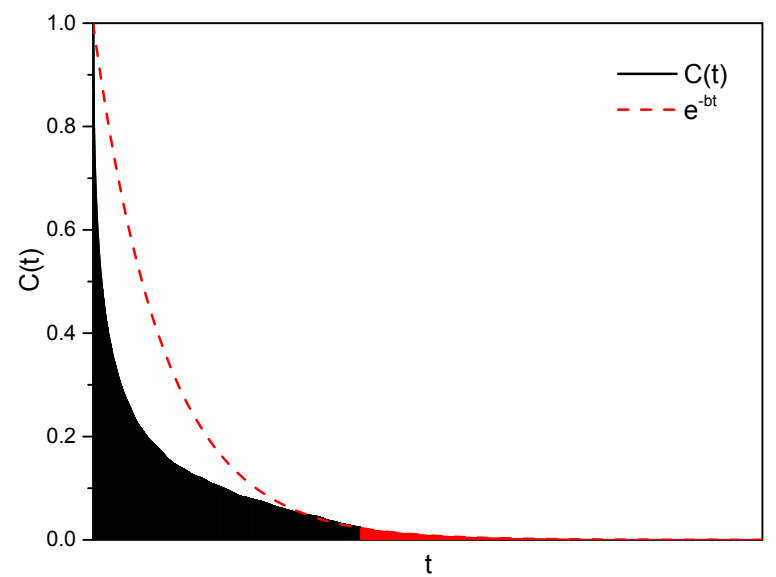

Figure S3. Schematic of the approximation of the time integral of correlation function.

$C(t)=e^{-b t}, \quad t>t_{0}$

$C\left(t_{0}\right)=e^{-b t_{0}}$

Then,

$\tau=\int_{0}^{\infty} C(t) d t \approx \int_{0}^{t_{0}} C(t) d t+\int_{t_{0}}^{\infty} e^{-b t} d t=\int_{0}^{t_{0}} C(t) d t+C\left(t_{0}\right) \frac{\int_{0}^{t_{0}} e^{-b t} d t}{1-C\left(t_{0}\right)}$

Because the $\int_{t_{0}}^{\infty} e^{-b t} d t$ is small, so we assume that

$\int_{0}^{t_{0}} e^{-b t} d t \approx \int_{0}^{t_{0}} C(t) d t$

Therefore, $\tau=\int_{0}^{\infty} C(t) d t \approx \frac{\int_{0}^{t_{0}} C(t) d t}{1-C\left(t_{0}\right)}$. 


\section{REFERENCES}

1. Shang, D. W.; Zhang, X. P.; Zeng, S. J.; Jiang, K.; Gao, H. S.; Dong, H. F.; Yang, Q. Y.; Zhang, S. J., Protic ionic liquid [Bim][NTf2] with strong hydrogen bond donating ability for highly efficient ammonia absorption. Green Chemistry 2017, 19, (4), 937-945.

2. Shang, D.; Bai, L.; Zeng, S.; Dong, H.; Gao, H.; Zhang, X.; Zhang, S., Enhanced NH3 capture by imidazolium - based protic ionic liquids with different anions and cation substituents. Journal of Chemical Technology \& Biotechnology 2018, 93, (5), 1228-1236.

3. Salgado, J.; Regueira, T.; Lugo, L.; Vijande, J.; Fernández, J.; García, J., Density and viscosity of three (2,2,2-trifluoroethanol + 1-butyl-3-methylimidazolium) ionic liquid binary systems. $J$ Chem Thermodyn 2014, 70, (5), 101-110.

4. Almeida, H. F. D.; Lopes, J. N. C.; Rebelo, L. P. N.; Coutinho, J. A. P.; Freire, M. G.; Marrucho, I. M., Densities and viscosities of mixtures of two ionic liquids containing a common cation. Journal of Chemical \& Engineering Data 2016, 61, (8), 2828-2843.

5. Vranes, M.; Dozic, S.; Djeric, V.; Gadzuric, S., Physicochemical Characterization of 1-Butyl-3methylimidazolium and 1-Butyl-1-methylpyrrolidinium Bis(trifluoromethylsulfonyl)imide. Journal of Chemical \& Engineering Data 2012, 57, (4), 1072-1077.

6. Liu, Q.; Zhao, L.; Zheng, Q.; Mou, L.; Zhang, P., Excess Molar Volume and Viscosity Deviation of [C2mim][NTf2]/[C4mim][NTf2]+ DMC/DEC. Journal of Chemical \& Engineering Data 2018, 63, (12), 4484-4496.

7. Tokuda, H.; Tsuzuki, S.; Susan, M. A. B. H.; Hayamizu, K.; Watanabe, M., How ionic are roomtemperature ionic liquids? An indicator of the physicochemical properties. The Journal of Physical Chemistry B 2006, 110, (39), 19593-19600.

8. Yadav, A.; Guha, A.; Pandey, A.; Pal, M.; Trivedi, S.; Pandey, S., Densities and dynamic viscosities of ionic liquids having 1-butyl-3-methylimidazolium cation with different anions and bis (trifluoromethylsulfonyl) imide anion with different cations in the temperature range (283.15 to 363.15) K. The Journal of Chemical Thermodynamics 2018, 116, 67-75.

9. Kassim, M. A.; Sairi, N. A.; Yusoff, R.; Ramalingam, A.; Alias, Y.; Aroua, M. K., Experimental densities and viscosities of binary mixture of 1-butyl-3-methylimidazolium bis (trifluoromethylsulfonyl) imide or glycerol with sulfolane and their molecular interaction by COSMO-RS. Thermochim Acta 2016, 639, 130-147.

10. Liu, H.; Maginn, E.; Visser, A. E.; Bridges, N. J.; Fox, E. B., Thermal and transport properties of six ionic liquids: an experimental and molecular dynamics study. Ind Eng Chem Res 2012, 51, (21), 7242-7254.

11. Fatima, U.; Anwar, N.; Montes-Campos, H.; Varela, L. M., Molecular dynamic simulation, molecular interactions and structural properties of 1-butyl-3-methylimidazolium bis (trifluoromethylsulfonyl) imide+ 1-butanol/1-propanol mixtures at (298.15-323.15) K and 0.1 M Pa. Fluid Phase Equilibria 2018, 472, 9-21.

12. Yasmeen, S.; Anwar, N., Interaction of 1-butyl-3-methylimidazolium bis (trifluoromethylsulfonyl)imide with methanol/dimethyl sulfoxide at $(298.15,303.15,308.15,313.15,318.15$ and 323.15$) \mathrm{K}$ : measurements and correlations of thermophysical properties. Journal of Molecular Liquids 2016, 221, 1207-1217.

13. Sha, M.; Dong, H.; Luo, F.; Tang, Z.; Zhu, G.; Wu, G., Dilute or Concentrated Electrolyte Solutions? Insight from Ionic Liquid/Water Electrolytes. $J$ Phys Chem Lett 2015, 6, (18), 3713-3720. 